\title{
Hippocampal networks habituate as novelty accumulates
}

\author{
Vishnu P. Murty, ${ }^{1,2,3}$ lan C. Ballard, ${ }^{1}$ Katherine E. Macduffie, ${ }^{4}$ Ruth M. Krebs, ${ }^{5}$ \\ and R. Alison Adcock ${ }^{1,2,4,6,7}$ \\ ${ }^{1}$ Center for Cognitive Neuroscience, Duke University, Durham, North Carolina 27708, USA; ${ }^{2}$ Department of Neurobiology, Duke \\ University, Durham, North Carolina 27708, USA; ${ }^{3}$ Department of Psychology, New York University, New York City, New York 10012, \\ USA; ${ }^{4}$ Department of Psychology and Neuroscience, Duke University, Durham, North Carolina 27708, USA; ${ }^{5}$ Department of \\ Experimental Psychology, Ghent University, 9000 Ghent, Belgium; ${ }^{6}$ Department of Psychiatry and Behavioral Sciences, Duke \\ University, Durham, North Carolina 27708, USA
}

\begin{abstract}
Novelty detection, a critical computation within the medial temporal lobe (MTL) memory system, necessarily depends on prior experience. The current study used functional magnetic resonance imaging (fMRI) in humans to investigate dynamic changes in MTL activation and functional connectivity as experience with novelty accumulates. fMRI data were collected during a target detection task: Participants monitored a series of trial-unique novel and familiar scene images to detect a repeating target scene. Even though novel images themselves did not repeat, we found that fMRI activations in the hippocampus and surrounding cortical MTL showed a specific, decrementing response with accumulating exposure to novelty. The significant linear decrement occurred for the novel but not the familiar images, and behavioral measures ruled out a corresponding decline in vigilance. Additionally, early in the series, the hippocampus was inversely coupled with the dorsal striatum, lateral and medial prefrontal cortex, and posterior visual processing regions; this inverse coupling also habituated as novelty accumulated. This novel demonstration of a dynamic adjustment in neural responses to novelty suggests a similarly dynamic allocation of neural resources based on recent experience.
\end{abstract}

A fundamental task for organisms is to detect, learn about, and respond to change in the environment. Novelty responses in the brain signal environmental change and predict neural and behavioral adjustments to it; neural responses to novelty are thus a proxy for the salience of environmental change. Evidence from humans, nonhuman primates, and rodents points to a specialized brain system for the detection of novelty, centered around the hippocampus (HPC) and medial temporal lobe (MTL) memory system (Ranganath and Rainer 2003). It is now well documented that the HPC and MTL respond robustly to novel stimuli (Gabrieli et al. 1997; Jessen et al. 2002; Kohler et al. 2005; Bunzeck and Duzel 2006; Yassa and Stark 2008; Blackford et al. 2010; Howard et al. 2011). However, relatively little is known about how these responses are modulated by prior experience. In particular, how does a recent history rich with novel information influence MTL networks specialized for novelty processing?

Other literature has described how prior exposure to biologically salient stimuli (or to a repeated feature) changes neural responses to future processing of those stimuli. Throughout the ventral visual stream, neural responses to repeated presentations of visually identical stimuli progressively decrease (Henson et al. 2003). Similarly, the amygdala and other structures implicated in fear processing habituate to cumulative exposure to trialunique stimuli that depict fear (Breiter et al. 1996; Wright et al. 2001; Fischer et al. 2003) or signal threat (Buchel et al. 1998; LaBar et al. 1998). Habituation has been proposed to be biologically adaptive: As a stimulus or feature is repeated, it provides less information about the environment, and demands fewer processing resources (Sokolov 1963; Rankin et al. 2009).

What if it is not a single stimulus or feature that is repeated but rather novelty itself? The biological salience of novelty should depend on an individual's history and current context; if so, sys-

\footnotetext{
${ }^{7}$ Corresponding author

E-mail alison.adcock@duke.edu

Article is online at http://www.learnmem.org/cgi/doi/10.1101/lm.029728.112.
}

tems specialized for novelty detection, like the MTL, should adapt to changes in the salience of novelty. Previous research shows that the MTL does adapt to repeated presentations of a specific stimulus (Brozinsky et al. 2005; Rankin et al. 2009), as well as to a single novel environment (Leussis and Bolivar 2006). The HPC has also been demonstrated to rapidly habituate to rare presentations of novelty that constitute expectancy violations (Strange and Dolan 2001; Yamaguchi et al. 2004). However, it is presently unknown how the MTL and its larger network dynamically respond to accumulating exposure to novelty across successive trial-unique stimuli.

The current study used functional magnetic resonance imaging (fMRI) to investigate whether the MTL exhibits changes in blood oxygenation level-dependent (BOLD) contrast (hereafter, "activation") or connectivity with other regions that reflect incrementally accumulating experience of stimulus novelty. During fMRI, participants viewed trial-unique (i.e., nonrepeated) novel and familiar scene images while monitoring the series for repetitions of a single familiar target scene image (Fig. 1). We asked whether the HPC and the surrounding MTL selectively habituated as successive presentations of novel compared to familiar scenes accumulated. Then, we investigated how functional connectivity between the HPC and other regions changed in response to these successive presentations. This characterization of MTL signaling and connectivity has implications for how the MTL adaptively shapes the allocation of neural resources dedicated to novelty processing.

\section{Results}

\section{Behavioral analysis}

Familiarization task

Participants were repeatedly exposed to a set of 80 scene images during the prescan familiarization task, which were later used as familiar scene images during the scanned, target detection task. 
Pre-Study Familiarization Task (outside of the scanner)

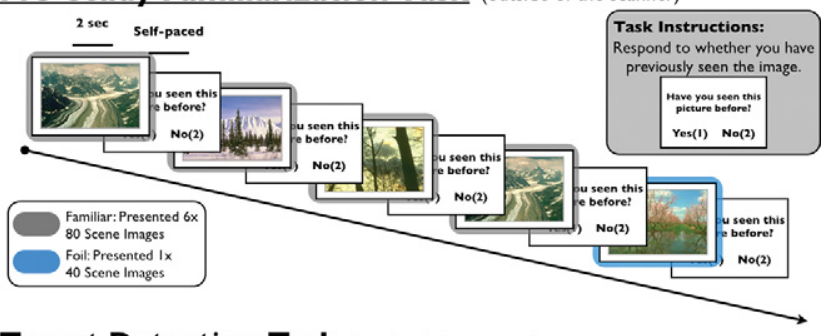

Target Detection Task (inside of the scanner)

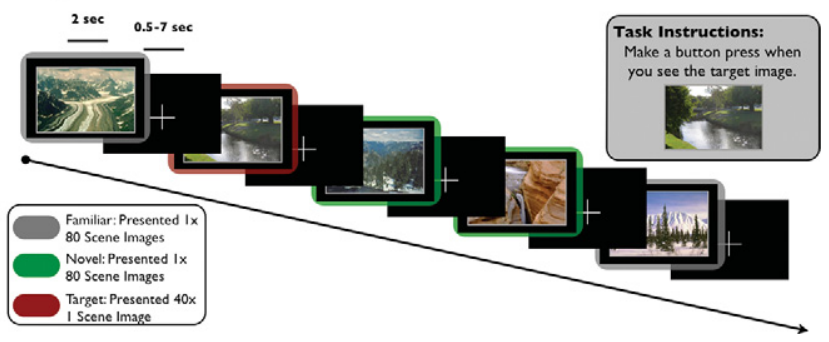

Figure 1. Experimental task. One hour prior to scanning, participants performed a prestudy familiarization task (top). During this task, participants viewed 80 outdoor scene images six times (familiar images) and 40 outdoor scene images once (foils), while responding whether they had previously seen each scene before. During fMRI scanning, participants performed a target detection task. During this task, participants made button presses to a target image, while incidentally viewing $80 \mathrm{fa}$ miliar scenes (which were presented during the prestudy familiarization task) and 80 novel images.

During this prestudy familiarization task, participants demonstrated significant corrected-recognition rates (hits-false alarms) to scene images on their sixth and final presentation (mean \pm $\left.\mathrm{SEM}=69.6 \% \pm 2.9 \%, t_{(23)}=24.0, P<0.001\right)$. These significant corrected-recognition rates indicate that prior to scanning participants were sufficiently familiar with the images that comprised the familiar scenes in the target detection task. Prior to the target detection task, participants performed two runs of an unrelated declarative memory paradigm that involved the presentation of novel object images; data from this task are not reported in this manuscript.

\section{Target detection task}

Target detection was nearly perfect across subjects (mean \pm $\mathrm{SEM}=99.4 \% \pm 0.3 \%$ ). Reaction times (RTs) to the target did not show significant variation over the course of the fMRI task $\left(F_{(3)}=1.539, P=0.21\right.$; linear trend, $P=0.23$; quadratic trend, $P=0.16)$. These RT results suggest that participants remained vigilant and engaged for the full duration of the task.

\section{fMRI analysis}

\section{Main effect of task (novel, familiar vs. baseline)}

To identify brain regions modulated by the presentation of novel and familiar scene images, we separately compared novel and familiar trials against the baseline fixation. Both novel and familiar scene images resulted in greater activations in HPC, perirhinal cortex (PRC), parahippocampal cortex (PHC), and throughout the ventral visual stream $(P<0.05$, whole-volume corrected $)$ (see Materials and Methods). Novel, but not familiar, trials elicited activation in the left head of the caudate $(P<0.05$, whole-volume corrected).

\section{Main effect of novelty (novel vs. familiar scenes)}

To identify brain regions differentially activated during the presentation of novel and familiar scene images, we directly contrasted activation in novel versus familiar trials. There were no regions that showed significantly greater activations to familiar compared to novel scene images. Compared to familiar scenes, the presentation of novel scenes resulted in significantly greater activation bilaterally in the PHC extending into the ventral visual stream and cerebellum $(P<0.05$, whole-volume corrected; left, $Z=3.47$, $[x, y, z]=[-28,-54,-14]$; right, $Z=4.69,[x, y, z]=[28,-40$, $-18]$ ) (Fig. 2). Notably, there were no significant activation differences in the HPC or caudate.

\section{Habituation of novelty responses (novel vs. familiar habituation)}

To identify brain regions that habituate differently to accumulating experience with successive trial-unique novel versus familiar scene images, we compared linearly decreasing activations on novel versus familiar trials. Greater habituation to novel compared to familiar scenes was observed bilaterally in the HPC, PRC, PHC, caudate, and throughout the ventral visual stream $(P<0.05$, whole-volume corrected) (Table $1 ;$ Fig. 3$)$. Further, significant habituation responses survived in the HPC, PRC, and dorsal striatum and in smaller portions of the PHC and ventral visual stream $(P<0.05$, whole-volume corrected) when we exclusively masked activation maps for the effect of familiar scene habituation at a liberal threshold of $P<0.05$, uncorrected (note that a more liberal threshold for an exclusive masks yields a more stringent final statistical map). This latter analysis ensures that habituation in these regions was predominantly driven by habituation to novel stimuli. There were no regions that showed significantly greater habituation to trial-unique presentations of familiar compared to novel scene images. It should be emphasized that these habituation responses to trial-unique stimuli are different from repetition suppression responses (Henson et al. 2003), which are responses to repeated presentations of identical stimuli.

To further characterize the nature of this habituation, we extracted single-trial estimates of HPC activations to novel and familiar scenes from the cluster identified in the analysis described above (see General Linear Model: Single-Trial Hippocampal Modeling, below, for details). We conducted post-hoc analyses to rule out the possibility that the declining contrast reflected incrementing activation to familiar scenes, rather than decrementing activation to novel scenes, and to rule out the rapid orienting response described in contextual violation paradigms (but see Strange and Dolan 2001; Yamaguchi et al. 2004). We found that the rate of habituation was characterized by a linear decrease for novel, but not familiar, scenes over time (novel, $F_{(1)}=11.92$, $P=0.002$; familiar, $F_{(1)}=1.02, P=0.32$ ). The linear decrease was not explained by changes in RTs; RTs showed no significant trends in the group and were uncorrelated with the hippocampal

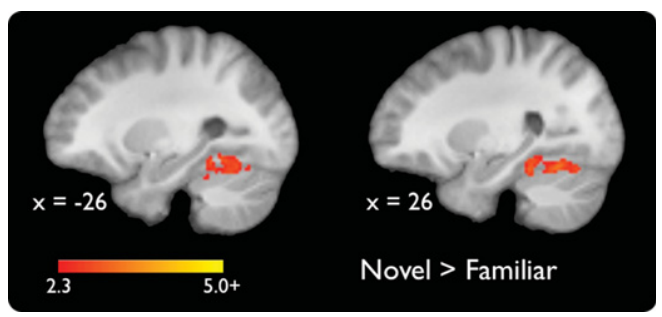

Figure 2. Significant activations for a contrast of novel versus familiar scenes $(P<0.05$, whole-volume corrected). Coordinates are in $\mathrm{MNI}$ space. 
Table 1. Regions showing significantly greater habituation to novel compared to familiar scenes $(P<0.05$, whole-volume corrected)

\begin{tabular}{lccc}
\hline Region & Voxel & Z-stat & $\begin{array}{c}\text { Z-max MNI } \\
\text { coordinates }(x, y, z)\end{array}$ \\
\hline $\begin{array}{l}\text { Bilateral hippocampus, } \\
\text { parahippocampal cortex, } \\
\text { perirhinal cortex, ventral } \\
\text { visual stream }\end{array}$ & 10932 & 5.77 & $(-30,-62,-18)$ \\
$\begin{array}{l}\text { Bilateral anterior cingulate, } \\
\text { corpus callosum }\end{array}$ & 269 & 3.53 & $(-10,28,2)$ \\
$\begin{array}{l}\text { R caudate } \\
\text { L caudate }\end{array}$ & 251 & 3.3 & $(18,-12,26)$ \\
\hline
\end{tabular}

The region column includes both peak voxels and other local maxima within a given cluster. The first region listed is the peak, while all following regions are other local maxima that exist in the cluster.

novelty decline at the individual level $(R=-0.02)$. There were no quadratic relationships with time for either condition; the adaptation of hippocampal responses to novelty was thus inconsistent with a rapid orienting response, instead declining linearly throughout the entire task (novel, $F_{(1)}=0.56, P=0.46$; familiar, $F_{(1)}=1.93$, $P=0.18)$.

\section{Changes in hippocampal connectivity during novelty processing}

To examine the influence of hippocampal habituation to novelty on the broader functional network, we used psychophysiological interaction (PPI) to identify regions whose connectivity with the HPC (in the cluster identified above) decreased with accumulating exposure to novel scenes. There was no habituation of positive coupling with the HPC. Habituation of negative coupling of the HPC was seen in the following regions: bilateral caudate, medial prefrontal cortex (PFC), cerebellum, ventral visual stream, and left lateral PFC, left putamen, left middle temporal gyrus, and left superior temporal gyrus $(P<0.05$, whole-volume corrected) (Fig. 4; Table 2).

\section{Discussion}

We investigated how the HPC and the surrounding cortical MTL adapt to successive novel events in a context where novelty is consistent and becomes expected. We found that HPC, along with the PRC, PHC, dorsal striatum, and ventral visual stream, showed a linear decrement, gradually habituating to accumulating experience with novelty. Our connectivity analysis indicated that upon early exposure to novelty the HPC was inversely coupled with the dorsal striatum, lateral PFC, medial PFC, and posterior visual processing regions; however, this inverse coupling also habituated. These findings demonstrate a neural proxy for changes in the salience of novelty over the course of experience, and suggest that the allocation of neural resources to future novelty processing reflects recent experience with novelty.

\section{Novelty processing in the MTL}

The HPC and surrounding MTL cortex are critical for processing novelty. Both rodent and human HPC lesions result in deficits in novelty-related behaviors (Ranganath and Rainer 2003) and human neuroimaging demonstrates engagement of these regions during novelty processing (Stern et al. 1996; Gabrieli et al. 1997; Jessen et al. 2002; Kohler et al. 2005; Bunzeck and Duzel 2006; Yassa and Stark 2008; Blackford et al. 2010; Howard et al. 2011). In our study, we found that novelty-related engagement of the
HPC and surrounding cortical MTL was dynamic: MTL novelty responses were greater at the beginning of the experiment and slowly declined as individuals gained experience with novelty. These habituation responses were specific to a linear decline in response to novelty, and did not interact with other components of our task. Specifically, our single-trial analysis indicated the linear decline was specific to the processing of novel scenes and did not occur during the processing of familiar scenes, and that no quadratic declines were present in either condition. Further, analysis of behavior during the target detection task revealed no linear or quadratic drifts in target RTs and no relationship to habituation across individuals, suggesting that our habituation results are not due to declining vigilance. Thus, our results suggest that the MTL and its extended network continuously integrate over past and present to determine the current salience of novelty.

We propose that this pattern of MTL novelty signaling reflects a biologically adaptive mechanism that dynamically matches neural processing resources to the expected salience of a given environmental input. Previous research has suggested that novelty indicates environmental change and thus is intrinsically salient. However, if individuals are exposed to novelty in the absence of behaviorally relevant outcomes, as they were in our current study, the salience of novelty and the associated neurophysiological response should habituate (Sokolov 1963). As noted above, previous studies have demonstrated habituation of the HPC in prolonged exposure to a single novel environment (Wilson and Rolls 1990a,b; Acquas et al. 1996; Giovannini et al. 2001) or repeated exposures to the same stimuli (Henson et al. 2003), as well as fear-predicting and fear-evoking stimuli (Buchel et al. 1999; Fischer et al. 2003). Previous research has also demonstrated that HPC responses are sensitive to experimentally instructed expectations about the value of information (Adcock et al. 2006; Murty et al. 2012) that should affect the salience of novelty (Wittmann et al. 2007; Bunzeck et al. 2010). Further, HPC novelty responses are greater when novelty itself is task-relevant (Stern et al. 2001; Hasselmo and Stern 2006). Together these previous lines of work provide evidence that the HPC has the capacity to integrate information about the salience of previously encountered novel events and to use that information to modulate current novelty responses. Within our current design, we only investigated changes in novelty responses in a context where their salience was expected to decrease. Future studies will need to investigate how manipulating the salience of novelty over time (i.e., by changing its task relevance) influences the pattern of HPC novelty responses.

Most relevant to our current findings are two prior demonstrations of rapid habituation of HPC responses to novelty (Strange and Dolan 2001; Yamaguchi et al. 2004). In both of these studies, HPC novelty signals habituated rapidly, as opposed to the linear, incremental decline we observed. Interestingly, in both of these studies, the novel stimuli were always oddballs (i.e., stimuli that are presented infrequently), thus violating participant

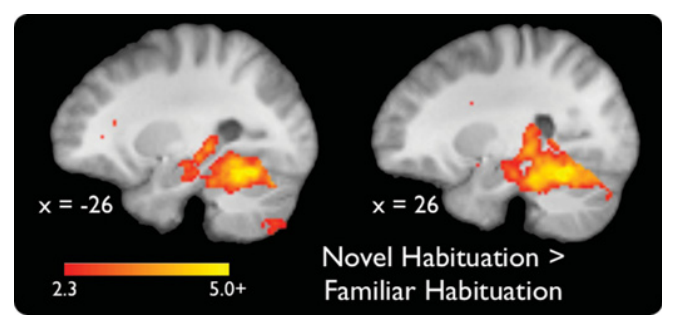

Figure 3. Significant activations for a contrast of habituation to novel versus familiar scenes $(P<0.05$, whole-volume corrected). Coordinates are in MNI space. 
Habituation of Novelty-Evoked Hippocampal Coupling

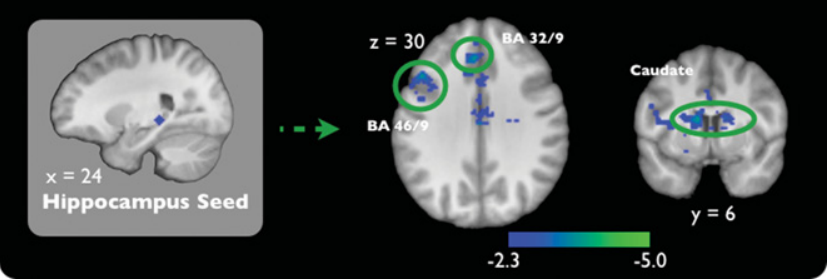

Figure 4. Brain regions showing significant habituation of functional connectivity with the right hippocampus $([x, y, z]=[24,-32,-3])$ during the presentation of novel scenes $(P<0.05$, whole-volume corrected). Coordinates are in MNI space.

expectations. Because the HPC is especially sensitive to expectancy violations (Ranganath and Rainer 2003; Kumaran and Maguire 2006), we propose that the rapid habituation seen in the previous studies reflects the magnitude of the expectancy violation. When novelty also presents an expectancy violation, the HPC should habituate rapidly, because rare novelty becomes expected as soon as participants orient to the structure of task. In the current study, both novel and familiar scenes were potential contextual or expectancy violations at the beginning of the task, yet no rapid declines were observed. We propose that our observed habituation responses reflect a slower and mechanistically distinct adaptation to the intrinsic salience or value of novelty. Future studies should investigate how these two forms of adaptation to novelty-task expectancy versus intrinsic value-interrelate within a single task design.

\section{Hippocampal connectivity during habituation to novelty}

Accumulating exposure to novelty also influenced hippocampal network connectivity: Although there were no differences in positive coupling, multiple exposures to novelty resulted in habituation of inverse coupling of the HPC with the dorsal striatum, lateral PFC, medial PFC, and posterior visual processing regions. These findings demonstrate that early exposures to novelty result in an inverse relationship between the HPC and the aforementioned regions; however, upon successive exposure to novelty this relationship diminishes. These findings emerged from a PPI analysis, which allowed us to address HPC coupling while statistically controlling for overall HPC activation. Thus, our connectivity findings are not explained by HPC activation alone, but rather reflect coordinated differences in HPC coupling with other neural regions.

We found changes in connectivity with both the lateral and medial PFC; however, we propose these regions differentially interact with hippocampal novelty processing. Specifically, we propose that the medial PFC contributes to hippocampal valuation of novelty's biological salience, as this region has been implicated in the assessment of environmental novelty and the behavioral ramifications of its presence (Rangel and Hare 2010; Grabenhorst and Rolls 2011; Schoenbaum et al. 2011; Silvetti et al. 2011; Roy et al. 2012). The medial PFC and other regions in the MTL, critically, predict habituation and extinction of behavioral responses to other biologically salient stimuli, such as fear-predicting stimuli (Vertes 2006; Schiller and Delgado 2010). Conversely, we propose that the lateral PFC manifests the downstream effects of novelty on cognition. Specifically, we propose that interactions between HPC and lateral PFC reflect an antagonistic relationship between HPC novelty signaling and lateral PFC goal-oriented processing. Task-irrelevant, novel stimuli have been demonstrated to disrupt lateral PFC-mediated, goal-oriented behaviors (Levy and
Goldman-Rakic 2000; Yantis 2000; Owen et al. 2005; Savine and Braver 2010; Ballard et al. 2011). Given that in this study novel scenes were task irrelevant, HPC responses to novel stimuli could potentially disrupt task-oriented processes for target detection within the lateral PFC. However, as novelty's salience diminishes, MTL responses in turn should habituate and the antagonistic relationship between these regions should decrease. These proposals offer hypotheses to be tested by future studies.

We also demonstrated a gradually habituating inverse relationship between the HPC and the dorsal striatum, specifically the caudate and putamen. Previous studies of associative learning have shown a trade-off between the MTL and striatum over time (Foerde and Shohamy 2011; Poldrack et al. 2001; White and McDonald 2002; Shohamy 2011) along with decreasing hippocampal activation (Amso et al. 2005); the dominant interpretation of these prior findings has been that they reflect the acquisition of either stimulus-response or stimulus-stimulus associations. We here demonstrate a similar antagonism between the striatum and HPC in a behavioral context devoid of explicit associative learning. Additionally, we show that this antagonistic relationship habituates over successive exposures to novelty, suggesting that as hippocampal sensitivity to novelty decreases, so does the bias away from striatal-based processing. Future studies are needed to address how these changes in HPC-striatum interactions influence memory encoding and other related behaviors.

\section{Future avenues of research}

The current study provides a novel characterization of MTL behavior in response to accumulating experience of novelty. The demonstration of this novel neural phenotype opens many questions regarding the mechanisms of this habituation and its consequences for behavior.

As described above, we propose a mechanism whereby the salience of novelty habituates in the absence of behaviorally relevant environmental change or consequence, following prior models of habituation (Sokolov 1963). Cognitive mechanisms, such as attention to novelty or interference from earlier novel stimuli with similar content, could be interacting with novelty to guide the habituation of HPC responses (although the stable RT data and the fact that many similar images were viewed prior to the beginning of the task argue against these examples as

Table 2. Regions showing habituation of functional connectivity with the right hippocampus $([x, y, z]=[24,-32,-3])$ during the presentation of novel scenes $(P<0.05$, whole-volume corrected)

\begin{tabular}{lllc}
\hline Region & Voxel & Z-stat & $\begin{array}{c}\text { Z-max MNI } \\
\text { coordinates }(x, y, z)\end{array}$ \\
\hline $\begin{array}{c}\text { Bilateral caudate, medial } \\
\text { frontal cortex (BA 32), } \\
\text { thalamus and L } \\
\text { dorsolateral prefrontal } \\
\quad \text { cortex (BA 9/46) }\end{array}$ & 3657 & 3.81 & $(-10,6,16)$ \\
$\begin{array}{c}\text { R cerebellum, ventral visual } \\
\text { stream }\end{array}$ & 698 & 4.21 & $(38,-72,-22)$ \\
$\begin{array}{c}\text { L cerebellum, ventral visual } \\
\text { stream }\end{array}$ & 289 & 3.81 & $(-52,-66,-26)$ \\
$\begin{array}{c}\text { L ventrolateral cortex (BA } \\
\quad 47), \text { putamen }\end{array}$ & 202 & 3.22 & $(-20,16,-14)$ \\
$\begin{array}{c}\text { L middle temporal gyrus (BA } \\
\text { 22), superior temporal } \\
\text { gyrus (BA 22) }\end{array}$ & 177 & 3.57 & $(-66,-34,-2)$ \\
\hline
\end{tabular}

The region column includes both peak voxels and other local maxima within a given cluster. The first region listed is the peak, while all following regions are other local maxima that exist in the cluster. 
primary explanations). Nonetheless, such possibilities are congruent with our interpretation, and offer interesting hypotheses for future studies to discern the cognitive mechanisms guiding this neural phenomenon.

The current study was not designed to investigate the impact of these neural-response patterns on behavior. Previous research has demonstrated reliable activation of the HPC and surrounding MTL during successful declarative memory encoding (Spaniol et al. 2009; Kim 2011), and models of declarative memory have proposed that novelty responses within the MTL are a determinant of successful encoding (Kirchhoff et al. 2000; Ranganath and Rainer 2003). Given these findings, our data make clear predictions regarding how accumulating exposure to novelty would influence memory encoding. Specifically, our data suggest that memory encoding for novel scenes would be greatest during initial exposures to novelty, but as HPC novelty signals habituate declarative memory, encoding for novel scenes would also begin to decline. Future behavioral and neuroimaging studies will need to specifically investigate what role these habituating signals in the HPC have in successful memory encoding.

\section{Summary}

The present study characterized changes in MTL activation and functional connectivity in response to accumulating experience with novelty. We found that HPC, cortical MTL, dorsal striatum, and ventral visual stream habituated to multiple presentations of trial-unique, novel scenes. Additionally, experience with novelty resulted in changes in hippocampal network connectivity, such that novelty-evoked inverse coupling of the HPC with the lateral PFC, medial PFC, and dorsal striatum also habituated. These findings demonstrate that MTL novelty signaling and connectivity are sensitive to the prior experience of novelty. Thus, as experience accumulates, novelty recruits fewer neural processing resources in the MTL and dynamically adjusts HPC network connectivity. This process does not reflect a rapid orientation to potential expectancy violations, but rather an incremental and continuous adjustment of the salience of novelty. We propose that the medial PFC guides this dynamic assessment of novelty's biological salience. Finally, we propose that these habituation processes may have downstream effects on systems guiding learning, decisionmaking, and executive functions by modulating HPC connectivity with the lateral PFC and dorsal striatum.

\section{Materials and Methods}

\section{Participants}

Twenty-eight healthy, right-handed participants were paid $\$ 40$ to participate, plus variable monetary bonuses earned during other tasks performed during the experimental session. All participants gave written informed consent for a protocol approved by the Duke University Institutional Review Board. Four participants were excluded: one for excessive head motion $(>1.5 \mathrm{~mm})$, one for poor performance during familiarization (chance performance), and two for computer malfunction. The final analyses included 24 participants (13 female, age range $=19-35$; median age $=25$ ).

\section{Procedures}

One hour prior to scanning, participants performed a task to familiarize themselves with 80 outdoor scene images (Fig. 1, top). During this task, participants viewed one outdoor scene image at a time (duration $2 \mathrm{sec}$ ) followed by a screen asking, "Have you seen this picture before?" Participants viewed each of the 80 outdoor scene images six times (familiar images) and 40 outdoor scene images once (foils) in a randomized order. This familiariza- tion task lasted $\sim 20 \mathrm{~min}$. We instructed participants to respond "yes/recognize" by pressing the " 1 " button and to respond "no/ new" by pressing the " 2 " button. Inside the scanner, prior to the target detection task, participants performed two runs of a declarative memory paradigm that involved the presentation of novel object images; data from this task are not reported in this manuscript.

Participants were instructed on the target detection task prior to scanning and again immediately prior to performing it (Fig. 1, bottom). We informed participants that during the task they would be presented with a series of outdoor scene images. We then showed them a single target scene image and instructed them to respond with a button press every time that target was presented. During each trial of the target detection task (Fig. 1, bottom), participants viewed an outdoor scene image (2 sec) followed by a fixation cross $(0.5-7 \mathrm{sec})$. Over the course of the task (duration $12 \mathrm{~min}, 2 \mathrm{sec}$ ), participants viewed 80 nonrepeated, novel scene images (images participants had never seen before), 80 trial-unique, familiar scene images (previously viewed during the prescan familiarization task), and 40 repetitions of the target scene image. Three methodological details are of note. First, we did not instruct participants that any of the scenes they viewed prior to scanning would appear again during this task; thus, we communicated no explicit expectations regarding novelty or familiarity for the target detection task. Second, the task described in the current study was preceded by a task in which no familiar images were shown, suggesting that there was no habituation to familiar stimuli prior to the start of the task. Third, individual novel and familiar scene images were shown only once each during the target detection task.

For both the familiarization and target detection task, scene images were all outdoor landscapes with a resolution of 100 pixels/inch. We randomly divided scene images into two sets and counterbalanced the sets between novel and familiar conditions across participants. We optimized trial order and onsets using Opt-seq software (Dale 1999). The order of novel and familiar trials was also counterbalanced across participants.

\section{MRI data acquisition and preprocessing}

fMRI data was acquired on a 3.0 T GE Signa MRI scanner using a standard echo-planar imaging (EPI) sequence $(\mathrm{TE}=27 \mathrm{msec}$, flip $=77^{\circ}, \mathrm{TR}=1 \mathrm{sec}, 17$ contiguous slices, size $3.75 \mathrm{~mm} \times 3.75$ $\mathrm{mm} \times 3.80 \mathrm{~mm}$ ). Partial brain data were acquired with a short TR in order to maximize the data sampling rate in regions of interest. Thus, fMRI data were acquired in the medial temporal lobe, striatum, medial frontal cortex, ventrolateral prefrontal cortex, and dorsolateral PFC, but excluded the frontopolar, orbitofrontal, motor, parietal, and primary visual cortices as well as the dorsal and posterior portions of the ventral visual stream. Data for the target detection task consisted of one run of 722 volumes. Prior to the functional run, we collected a whole-brain, inversion recovery, spoiled gradient recalled (IR-SPGR) high-resolution anatomical image (voxel size $=1 \mathrm{~mm}$, isotropic) for spatial normalization and a whole-brain EPI image (34 contiguous slices) with the same voxel size and orientation as partial EPI brain images to assist with coregistration.

fMRI preprocessing was performed using fMRI Expert Analysis Tool (FEAT) Version 5.92 as implemented in FSL 4.1.5 (www.fmrib.ox.ac.uk/fsl). BOLD images were skull stripped, realigned, intensity normalized by a single multiplicative factor, spatially smoothed with a FWHM of $4.0 \mathrm{~mm}$ and subjected to a 100-sec, high-pass temporal filter. Spatial normalization was performed using a three-step fMRIb Linear Registration Tool (FLIRT) procedure. First, mean partial-volume EPIs were coregistered to the reference whole-brain EPI. Then the whole-brain EPI was coregistered to the high-resolution anatomical image. Finally, the high-resolution anatomical image was normalized to the high-resolution standard space image using a nonlinear transformation with a $10-\mathrm{mm}$ warp resolution as implemented by the fMRI Non-Linear Registration Tool (FNIRT). These transforms were then applied to the EPI data. All coordinates are reported in MNI space. 


\section{Behavioral analysis}

To confirm familiarization with scene images presented during the prescan familiarization task, we submitted corrected memory recognition scores for the final (sixth) presentation of each image to a one-sample $t$-test. Corrected recognition was defined as the percent of familiarized images endorsed as old (during the sixth presentation) subtracted by the percent of novel foils incorrectly endorsed as old. To measure performance during the scanned target detection task, we submitted target detection accuracy to a one-sample $t$-test. To indirectly assess potential attentional drifts across the target detection task, we submitted mean target RTs from quartiles of the experimental task to a repeated-measures ANOVA and tested for linear and quadratic trends. To rule out a relationship between RT and hippocampal activation, we also calculated each individual's rate of change in RT and tested for correlation with each individual's change in hippocampal parameter estimates.

\section{fMRI data analysis}

fMRI data were analyzed using FEAT Version 5.92 as implemented in FSL 4.1.5. Time-series statistical analyses used FILM with local autocorrelation correction (Woolrich et al. 2001).

\section{General linear model: novelty analysis}

To investigate the main effect of novelty, we modeled individualsubject data using fixed-effect general linear models (GLMs) with separate regressors for novel, familiar, and target conditions. Individual events were convolved with a double-gamma hemodynamic response (HDR) function (duration $=2 \mathrm{sec}$, input value $=$ 1). Using this GLM, individual maps of parameter estimates were generated for the contrasts of interest. These contrasts included novel scene images versus baseline, familiar scene images versus baseline, and novel scene images versus familiar scene images. (Presentations of the repeated target image were confounded with requirement for motor responses, limiting their utility for the novelty analysis.)

\section{General linear model: novelty habituation analysis}

To investigate whether novelty responses habituate as experience with novelty accumulates, we modeled individual-subject data using fixed-effect GLMs with three condition regressors and two parametric habituation regressors. Condition regressors separately modeled novel, familiar, and target conditions (duration = 2 sec, input value $=1$ ). Parametric habituation regressors separately modeled novel and familiar conditions with a 2-sec duration and input value that linearly decreased over trial number (i.e., experience with novel or familiar scenes). Specifically, parametric input values ranged from 1 to -1 and were highest for the first presentation of a scene and lowest for the last presentation of a scene within a condition (novel or familiar). Both standard and parametric regressors were convolved with a doublegamma HDR function. Using this GLM, individual parameter estimate maps were generated for the contrast of interest: Parametric decreases to novel scenes images versus parametric decreases to familiar scene images.

\section{General linear model: single-trial hippocampal modeling}

To further characterize the form of the hippocampal habituation to novelty, we conducted a single-trial analysis. We constructed separate GLMs modeling each novel and familiar event in isolation, while modeling all other events as nuisance regressors. This method of single-trial analysis has been demonstrated to better characterize the neural activations to a single event (for a detailed description of this method see Mumford et al. 2012). We then calculated the mean of the single-trial parameter estimates extracted across all voxels in the hippocampal cluster identified in the habituation analysis (see above), and submitted these parameter estimates to a repeated-measure ANOVA with condition and time as within-subjects factors. Condition included two lev- els, novel and familiar, and time included five levels, partitioning events into blocks of 16 temporally adjacent trials. We then tested for linear and higher-order trends across time and tested for a relationship of these trends with each individual's rate of change in RT at a significance threshold of $P<0.05$. This region of interest (ROI) approach should be more conservative for ruling out potential quadratic effects than including a quadratic term in the parametric GLM model of the fMRI data, because it does not require an arbitrarily chosen steepness for the quadratic parametric regressor. Instead, by analyzing the extracted $\beta$-parameters in an ANOVA, we were able to test the full model space of exponential responses.

\section{Psychophysiological interaction (PPI): hippocampal connectivity analysis}

To investigate habituation of hippocampal connectivity to novel scenes, we identified PPIs of novelty habituation and functional connectivity with the HPC. We modeled individual-subject data using fixed-effects GLMs with three condition regressors, two parametric habituation regressors, one physiological regressor, and two PPI regressors. Standard regressors to novel, familiar, and target conditions, and parametric regressors of habituation to novel and familiar scenes were modeled as in the "novelty habituation analysis." The physiological regressor was the first eigenvariate of a sphere (radius $=4 \mathrm{~mm}$ ) centered on the peak voxel derived from the contrast "Novelty Habituation versus Familiar Habituation" $([x, y, z]=[24,-32,-4])$ within the HPC (HPC defined using Harvard-Oxford Subcortical Structural Atlas). The PPI regressors were constructed by multiplying this HPC physiological regressor separately with (1) the standard regressor of novelty and (2) the novelty habituation regressor. Thus, the PPI novelty regressor modeled differences in hippocampal coupling across the brain as a function of novelty and habituation to novelty. Using this GLM, individual parameter estimate maps were generated for the contrast of interest: hippocampal connectivity during novel scenes that parametrically decreased upon accumulating exposure to novel scenes.

\section{General linear model: group-level analyses}

Group-level analyses, including the PPI analysis, were modeled using mixed-effects analyses (FLAME 1) on the parameter estimates derived from the contrasts of interest from the singlesubject GLMs. Whole-volume, group-level analyses were analyzed using a voxel significance threshold of $z>2.3$ and a clustersignificance threshold of $P<0.05$, fully corrected for all voxels in our imaging volume (Worsley 2001).

\section{Acknowledgments}

This project was supported by National Institutes of Health grants R01 DA027802 and R01 MH094743. We thank S. Davis and R.M. Carter for helpful discussions and comments.

\section{References}

Acquas E, Wilson C, Fibiger HC. 1996. Conditioned and unconditioned stimuli increase frontal cortical and hippocampal acetylcholine release: Effects of novelty, habituation, and fear. J Neurosci 16: 3089-3096.

Adcock RA, Thangavel A, Whitfield-Gabrieli S, Knutson B, Gabrieli JD. 2006. Reward-motivated learning: Mesolimbic activation precedes memory formation. Neuron 50: 507-517.

Amso D, Davidson MC, Johnson SP, Glover G, Casey BJ. 2005. Contributions of the hippocampus and the striatum to simple association and frequency-based learning. Neuroimage 27: 291-298.

Ballard IC, Murty VP, Carter RM, MacInnes JJ, Huettel SA, Adcock RA. 2011. Dorsolateral prefrontal cortex drives mesolimbic dopaminergic regions to initiate motivated behavior. J Neurosci 31: 10340-10346.

Blackford JU, Buckholtz JW, Avery SN, Zald DH. 2010. A unique role for the human amygdala in novelty detection. Neuroimage 50: 1188-1193.

Breiter HC, Etcoff NL, Whalen PJ, Kennedy WA, Rauch SL, Buckner RL, Strauss MM, Hyman SE, Rosen BR. 1996. Response and habituation of the human amygdala during visual processing of facial expression. Neuron 17: 875-887. 
Brozinsky CJ, Yonelinas AP, Kroll NE, Ranganath C. 2005. Lag-sensitive repetition suppression effects in the anterior parahippocampal gyrus. Hippocampus 15: 557-561.

Buchel C, Morris J, Dolan RJ, Friston KJ. 1998. Brain systems mediating aversive conditioning: An event-related fMRI study. Neuron 20: 947-957.

Buchel C, Dolan RJ, Armony JL, Friston KJ. 1999. Amygdala-hippocampal involvement in human aversive trace conditioning revealed through event-related functional magnetic resonance imaging. J Neurosci 19: 10869-10876.

Bunzeck N, Duzel E. 2006. Absolute coding of stimulus novelty in the human substantia nigra/VTA. Neuron 51: 369-379.

Bunzeck N, Dayan P, Dolan RJ, Duzel E. 2010. A common mechanism for adaptive scaling of reward and novelty. Hum Brain Mapp 31: 1380-1394

Dale AM. 1999. Optimal experimental design for event-related fMRI. Hum Brain Mapp 8: 109-114.

Fischer H, Wright CI, Whalen PJ, McInerney SC, Shin LM, Rauch SL. 2003. Brain habituation during repeated exposure to fearful and neutral faces: A functional MRI study. Brain Res Bull 59: 387-392.

Foerde K, Shohamy D. 2011. The role of the basal ganglia in learning and memory: Insight from Parkinson's disease. Neurobiol Learn Mem 96: 624-636.

Gabrieli JD, Brewer JB, Desmond JE, Glover GH. 1997. Separate neural bases of two fundamental memory processes in the human medial temporal lobe. Science 276: $264-266$.

Giovannini MG, Rakovska A, Benton RS, Pazzagli M, Bianchi L, Pepeu G. 2001. Effects of novelty and habituation on acetylcholine, GABA, and glutamate release from the frontal cortex and hippocampus of freely moving rats. Neuroscience 106: 43-53.

Grabenhorst F, Rolls ET. 2011. Value, pleasure and choice in the ventral prefrontal cortex. Trends Cogn Sci 15: 56-67.

Hasselmo ME, Stern CE. 2006. Mechanisms underlying working memory for novel information. Trends Cogn Sci 10: 487-493.

Henson RN, Cansino S, Herron JE, Robb WG, Rugg MD. 2003. A familiarity signal in human anterior medial temporal cortex? Hippocampus 13: 301-304.

Howard LR, Kumaran D, Olafsdottir HF, Spiers HJ. 2011. Double dissociation between hippocampal and parahippocampal responses to objectbackground context and scene novelty. I Neurosci 31: 5253-5261.

Jessen F, Manka C, Scheef L, Granath DO, Schild HH, Heun R. 2002. Novelty detection and repetition suppression in a passive picture viewing task: A possible approach for the evaluation of neuropsychiatric disorders. Hum Brain Mapp 17: 230-236.

Kim H. 2011. Neural activity that predicts subsequent memory and forgetting: A meta-analysis of 74 fMRI studies. Neuroimage 54: $2446-2461$.

Kirchhoff BA, Wagner AD, Maril A, Stern CE. 2000. Prefrontal-temporal circuitry for episodic encoding and subsequent memory. J Neurosci 20: $6173-6180$.

Kohler S, Danckert S, Gati JS, Menon RS. 2005. Novelty responses to relational and non-relational information in the hippocampus and the parahippocampal region: A comparison based on event-related fMRI. Hippocampus 15: 763-774.

Kumaran D, Maguire EA. 2006. An unexpected sequence of events: Mismatch detection in the human hippocampus. PLoS Biol 4: e424.

LaBar KS, Gatenby JC, Gore JC, LeDoux JE, Phelps EA. 1998. Human amygdala activation during conditioned fear acquisition and extinction: A mixed-trial fMRI study. Neuron 20: 937-945.

Leussis MP, Bolivar VJ. 2006. Habituation in rodents: A review of behavior neurobiology, and genetics. Neurosci Biobehav Rev 30: 1045-1064

Levy R, Goldman-Rakic PS. 2000. Segregation of working memory functions within the dorsolateral prefrontal cortex. Exp Brain Res 133: $23-32$.

Mumford JA, Turner BO, Ashby FG, Poldrack RA. 2012. Deconvolving BOLD activation in event-related designs for multivoxel pattern classification analyses. Neuroimage 59: 2636-2643.

Murty VP, Labar KS, Adcock RA. 2012. Threat of punishment motivates memory encoding via amygdala, not midbrain, interactions with the medial temporal lobe. J Neurosci 32: 8969-8976.

Owen AM, McMillan KM, Laird AR, Bullmore E. 2005. N-back working memory paradigm: A meta-analysis of normative functional neuroimaging studies. Hum Brain Mapp 25: 46-59.

Poldrack RA, Clark J, Pare-Blagoev EJ, Shohamy D, Creso Moyano J, Myers C, Gluck MA. 2001. Interactive memory systems in the human brain. Nature 414: $546-550$.
Ranganath C, Rainer G. 2003. Neural mechanisms for detecting and remembering novel events. Nat Rev Neurosci 4: 193-202.

Rangel A, Hare T. 2010. Neural computations associated with goal-directed choice. Curr Opin Neurobiol 20: 262-270.

Rankin CH, Abrams T, Barry RJ, Bhatnagar S, Clayton DF, Colombo J, Coppola G, Geyer MA, Glanzman DL, Marsland S, et al. 2009. Habituation revisited: An updated and revised description of the behavioral characteristics of habituation. Neurobiol Learn Mem 92: $135-138$.

Roy M, Shohamy D, Wager TD. 2012. Ventromedial prefrontal-subcortical systems and the generation of affective meaning. Trends Cogn Sci 16: $147-156$.

Savine AC, Braver TS. 2010. Motivated cognitive control: Reward incentives modulate preparatory neural activity during task-switching. J Neurosci 30: $10294-10305$

Schiller D, Delgado MR. 2010. Overlapping neural systems mediating extinction, reversal and regulation of fear. Trends Cogn Sci 14: 268-276.

Schoenbaum G, Takahashi Y, Liu TL, McDannald MA. 2011. Does the orbitofrontal cortex signal value? Ann N Y Acad Sci 1239: 87-99.

Shohamy D. 2011. Learning and motivation in the human striatum. Curr Opin Neurobiol 21: 408-414.

Silvetti M, Seurinck R, Verguts T. 2011. Value and prediction error in medial frontal cortex: Integrating the single-unit and systems levels of analysis. Front Hum Neurosci 5: 75.

Sokolov EN. 1963. Perception and the conditioned reflex. Pergamon Press, Oxford, UK.

Spaniol J, Davidson PS, Kim AS, Han H, Moscovitch M, Grady CL. 2009. Event-related fMRI studies of episodic encoding and retrieval: Meta-analyses using activation likelihood estimation. Neuropsychologia 47: $1765-1779$.

Stern CE, Corkin S, Gonzalez RG, Guimaraes AR, Baker JR, Jennings PJ, Carr CA, Sugiura RM, Vedantham V, Rosen BR. 1996. The hippocampal formation participates in novel picture encoding: Evidence from functional magnetic resonance imaging. Proc Natl Acad Sci 93: $8660-8665$.

Stern CE, Sherman SJ, Kirchhoff BA, Hasselmo ME. 2001. Medial temporal and prefrontal contributions to working memory tasks with novel and familiar stimuli. Hippocampus 11: 337-346.

Strange BA, Dolan RJ. 2001. Adaptive anterior hippocampal responses to oddball stimuli. Hippocampus 11: 690-698.

Vertes RP. 2006. Interactions among the medial prefrontal cortex, hippocampus and midline thalamus in emotional and cognitive processing in the rat. Neuroscience 142: 1-20.

White NM, McDonald RJ. 2002. Multiple parallel memory systems in the brain of the rat. Neurobiol Learn Mem 77: 125-184.

Wilson FA, Rolls ET. 1990a. Learning and memory is reflected in the responses of reinforcement-related neurons in the primate basal forebrain. J Neurosci 10: 1254-1267.

Wilson FA, Rolls ET. 1990b. Neuronal responses related to the novelty and familiarity of visual stimuli in the substantia innominata, diagonal band of Broca and periventricular region of the primate basal forebrain. Exp Brain Res 80: 104-120.

Wittmann BC, Bunzeck N, Dolan RJ, Duzel E. 2007. Anticipation of novelty recruits reward system and hippocampus while promoting recollection. Neuroimage 38: 194-202.

Woolrich MW, Ripley BD, Brady M, Smith SM. 2001. Temporal autocorrelation in univariate linear modeling of FMRI data. Neuroimage 14: $1370-1386$.

Worsley KJ. 2001. Statistical analysis of activation images. In Functional MRI: An introduction to methods (ed. Jezzard P, et al.), Vol 1, pp. 251-270. Oxford University Press, Oxford, UK.

Wright CI, Fischer H, Whalen PJ, McInerney SC, Shin LM, Rauch SL. 2001. Differential prefrontal cortex and amygdala habituation to repeatedly presented emotional stimuli. Neuroreport 12: $379-383$.

Yamaguchi S, Hale LA, D'Esposito M, Knight RT. 2004. Rapid prefrontal-hippocampal habituation to novel events. J Neurosci 24: $5356-5363$.

Yantis S. 2000. Attentional capture in vision. In Control of cognitive processes: Attention and performance XVIII (ed. Monsell S, Driver J), pp. 71-208. MIT Press, Cambridge, MA.

Yassa MA, Stark CE. 2008. Multiple signals of recognition memory in the medial temporal lobe. Hippocampus 18: 945-954.

Received November 20, 2012; accepted in revised form February 6, 2013. 


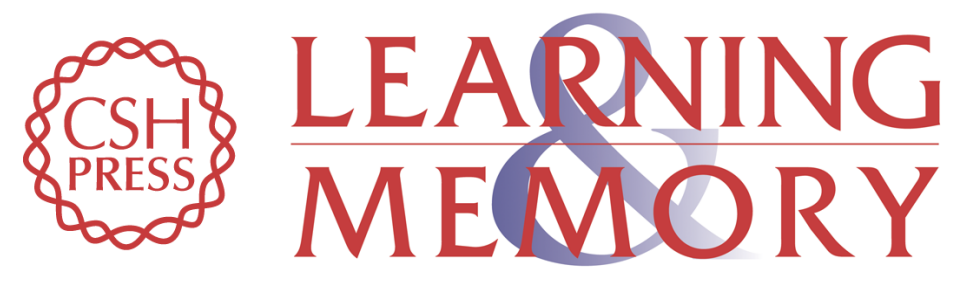

\section{Hippocampal networks habituate as novelty accumulates}

Vishnu P. Murty, Ian C. Ballard, Katherine E. Macduffie, et al.

Learn. Mem. 2013, 20:

Access the most recent version at doi:10.1101//m.029728.112

References This article cites 55 articles, 11 of which can be accessed free at: http://learnmem.cshlp.org/content/20/4/229.full.html\#ref-list-1

License

Email Alerting Receive free email alerts when new articles cite this article - sign up in the box at the Service top right corner of the article or click here. 